transient infections. We aimed to identify the population of adolescents infected with HPV and risk factors associated.

Methods A cross-sectional study evaluating adolescents (from 10 to 19 years of age) carried out from January to August 2012 at the major Sexually Transmitted Infections (STI) Reference Centre in Bahia, Brazil. Sociodemographic and clinical data were obtained by reviewing charts and analysed through SPSS 20.0 .

Results Of the adolescents evaluated, 324 had the diagnosis of HPV, evidencing an HPV prevalence of $62.5 \%$. Among those, the mean age was $16.8( \pm 1.5)$ years and the sexual debut occurred at $13.2( \pm 3.5)$ years of age. There was an association between the female gender and the diagnosis of HPV $(\mathrm{p}<0.01$; OR 3.22 , 95\% CI 2.18-4.77), as well as to being 16 years old or older $(\mathrm{p}<0.01$; OR 3.44 , 95\% CI $2.28-5.19)$. Regarding lifestyle, 25.6\% (42/164) of HPV patients reported alcohol use, 8.1\% (16/197) illicit drugs use and 2.2\% (3/138) were smokers. There was a statistical correlation between having $\geq 8$ years of schooling and HPV $(p=0.02$; OR 1.76, 95\% CI 1.10-2.79), as well as working and the infection $(p=0.01$; OR 2.54, 95\% CI 1.17-5.53). As for clinical characteristics, $15.8 \%(40 / 253)$ of HPV patients were pregnant during the study, with a significant association between pregnancy and the virus $(\mathrm{p}=0.02$; OR 2.69, 95\% CI 1.10-6.58). There was $91.9 \%$ (295/321) of clinical diagnosis of HPV, and $61.9 \%$ $(199 / 321)$ of genital warts among the infected population. $90.9 \%(280 / 308)$ of the infected adolescents underwent treatment for HPV.

Conclusion The clinical and epidemiological profile of these adolescents revealed the need for prevention campaigns against STIs with accessible language, promoting access to information. Access to HPV vaccine is now possible, and it is still necessary to stimulate follow-up and treatment in order to reduce this infection and its associated diseases.

\section{P3.186 NEISSERIA GONORRHOEAE ANTIMICROBIAL RESISTANCE SURVEILLANCE IN JOHANNESBURG, SOUTH AFRICA}

${ }^{1}$ Ranmini Kularatne, ${ }^{2}$ Venessa Maseko, ${ }^{2}$ Lindy Gumede, ${ }^{2}$ Frans Radebe, ${ }^{1}$ Tendesayi KufaChakezha. 'National Institute for Communicable Diseases and University of The Witwatersrand, Johannesburg, South African Republic; ${ }^{2}$ National Institute for Communicable Diseases, Johannesburg, South African Republic

\subsection{6/sextrans-2017-053264.421}

Introduction Gonorrhoea is the predominant cause of symptomatic male urethritis in South Africa. Neisseria gonorrhoeae has displayed an alarming propensity to acquire resistance to all sequential first-line antimicrobial agents used over the years. The National Institute for Communicable Diseases has co-ordinated STI microbiological surveillance since 2005 in order to validate existing national syndromic management guidelines. We describe Neisseria gonorrhoeae antimicrobial resistance patterns and trends from Johannesburg surveillance, spanning a period of eight years from 2008 to 2015 .

Methods Neisseria gonorrhoeae was cultured from swab specimens of genital discharge (endocervical and endourethral) from consenting adult patients presenting to a communitybased primary healthcare facility in Johannesburg. The minimum inhibitory concentrations (MICs) of antimcrobials were determined using Etest (cefixime, ceftriaxone, ciprofloxacin) or agar dilution (azithromycin, penicillin, tetracycline). Clinical Laboratory Standards Institute (CLSI) criteria, where applicable, were used for interpretation of results. Descriptive statistics and likelihood-ratio tests in STATA 14 were used for data analysis.

Results A total of 2,112 Neisseria gonorrhoeae isolates were tested for susceptibility to the extended-spectrum cephalosporins (ESCs) and ciprofloxacin. A proportion of these were tested for susceptibility to penicillin, tetracycline and azithromycin. Between 2011 and 2015, the prevalence of high-level resistance increased from $31 \%$ to $57 \%$ for penicillin $(p=0.009)$ and $73 \%$ to $91 \%$ for tetracycline $(p=0.009)$. Between 2008 and 2015, the prevalence of high-level ciprofloxacin resistance rose exponentially from $24 \%$ to $67 \%$ $(\mathrm{p}<0.001)$. Decreased susceptibility (DS) to cefixime was not observed; however one isolate from 2013 exhibited DS to ceftriaxone. Trend analysis revealed MIC creep for cefixime. Elevated azithromycin MICs of $>0.5 \mu \mathrm{g} / \mathrm{ml}$ were identified only in $2015(5 / 125 ; 4 \%)$.

Conclusion Neisseria gonorrhoeae resistance trends for Johannesburg reveal that high-prevalence resistance to penicillin, tetracycline and ciprofloxacin obviates their use for STI syndromic management. The prevalence of resistance to ESCs is $<1 \%$ and to azithromycin $<5 \%$, validating their continued use in dual therapy. However, it is essential that ESC and azithromycin susceptibility trends are monitored to detect emerging resistance timeously.

\section{P3.187 HIV AND STI PREVENTION AMONG YOUNG MEN WHO HAVE SEX WITH MEN (MSM) IN THE WESTERN REGION OF PUERTO RICO}

${ }^{1}$ Raquel Corchado, ${ }^{2}$ Vivian Colon, ${ }^{3}$ Edna Acosta, ${ }^{4}$ Jorge Rodríguez, ${ }^{5}$ Yara Sánchez, ${ }^{5}$ Nelson Castillo, ${ }^{6}$ Luis Nieves, ${ }^{7}$ Jose Mulinelli, ${ }^{8}$ Peter Shepard, ${ }^{7}$ Cesar Concepcion. ${ }^{1}$ Department of Social Sciences, Graduate School of Public Health, Medical Sciences Campus, Universit, San Juan - Puerto Rico; ${ }^{2}$ Population Sciences Division, Puerto Rico Comprehensive Cancer Centre, San Juan - Puerto Rico; 3 Community Services Division, Centre for Evaluation and Sociomedical Research, Graduate School of Publ, San Juan Puerto Rico; ${ }^{4}$ Department of Health Services Administration, Graduate School of Public Health, Medical Sciences CA, San Juan - Puerto Rico; ${ }^{5}$ Department of Biostatistics And Epidemiology, Graduate School of Public Health, Medical Sciences CA, San Juan - Puerto Rico; ${ }^{6}$ Department of Social Sciences, Mayagüez Campus, University of Puerto Rico, Mayagüez - Puerto Rico; ${ }^{7}$ Coaí INC, Mayagüez - Puerto Rico; 8. Coaí, INC, Mayagüez Puerto Rico

\subsection{6/sextrans-2017-053264.422}

Introduction According to the HIV Surveillance System, there has been an increase in newly reported cases of HIV among young men who have sex with men (YMSM) in Puerto Rico when compared to intravenous drug users. The Youth Prevention Program (YPREV) which aims to monitor high-risk practices in young men (18-24 years old) and provide services for this population in the Western region of the island was developed between the academia and community-based organisations (CBOs) with the aim of decreasing health disparities in youth in universities and surrounding communities. This study presents data on the first year of the needs assessment concerning HIV testing and prevention methods as well as our ongoing efforts after gathering this data.

Methods To determine the needs and outline the strategic plan of YPREV, a mixed methods approach was used. The first phase consisted of developing and carrying out a needs assessment which was performed from March 2016 until September 2016. A total of 183 YMSM were recruited using a 
convenience sampling. Descriptive analysis was used to portray reported prevention efforts for HIV among YMSM.

Results Our results showed that $36.3 \%$ of the participants reported never being tested for HIV. Among those never being tested, 28.6\% didn't know where to get tested, $27.1 \%$ did not want to use their parent's medical insurance and $17.1 \%$ feared of obtaining the results. An overwhelming 90.7\% reported that they were willing to get tested if the test were free. Most (73.1\%) reported never having been offered an HIV test by their health provider or CBO. $42.6 \%$ reported not knowing where they can obtain free condoms in campus or surrounding communities, while $35.3 \%$ of the participants who had sexual relations in the last 30 days with men reported not using a condom.

Conclusion YPREV intends to increase training activities for health providers regarding the importance of HIV testing and has implemented free monthly HIV testing clinics since September 2016. This program aims to strengthen prevention initiatives for young men at risk for HIV.

Support: The Youth Prevention Program is part the CCC and is funded by the Substance Abuse and Mental Health Service Administration (SAMHSA) grant \# 1H79SP021376.

\section{P3.188 RISK BEHAVIOURS IN HOMELESS PERSONS BASED ON HIV STATUS IN CENTRAL BRAZIL}

Raquel Silva Pinheiro, Paulie Marcelly Ribeiro Dos Santos Carvalho, Thaynara Lorrane Silva Martins, Bruno César Teodoro Martins, Camila Canhete Ferreira, Karlla Antonieta Amorin Caetano, Marcos André De Matos, Sheila Araújo Teles. Faculdade de Enfermagem da Universidade Federal de Goiás, Goiânia - GO, Brazil

\subsection{6/sextrans-2017-053264.423}

Introduction Mortality rates among the homeless are greater than the general population, presenting a high prevalence of comorbidities and exposure to risk factors. Housing insecurity has been associated with a high risk of sexual and physical exploitation and involvement in illicit activities as means of survival, contributing to the acquisition of sexually transmitted infections (STI), such as human immunodeficiency virus (HIV). The aim of this study, cross sectional, was to identify risk behaviours for infection by HIV among the homeless in Goiás, Central Brazil.

Methods Between September 2014 to August 2015, 356 individuals served by a public shelter in the City of Goiânia, Central Brazil, were interviewed and tested for HIV. Multivariable logistic regression model was used to identify variables associated with HIV infection.

Results Of the 356 recruited individuals, 81.2\% were male, young (median: 36 years), with a low level of education (median: 7 years of study), self-reported mixed race $(60.4 \%)$, single $(59.6 \%)$ and religious $(82.3 \%)$. Of the total, $3.9 \%$ (95\% CI: $2.2 \%-6.4 \%$ ) were HIV positive. Individuals infected by HIV reported greater risk behaviours, such as: homosexuality/bisexuality (35.7\%), consumption of illicit drugs (92.9\%), sexual relations with HIV carriers $(45.5 \%)$, and a history of STI $(61.5 \%)$ (all $\mathrm{p} \leq 0,05)$. Logistic regression analysis revealed that the number of sexual partners (adjusted odds ratio [AOR]: $6.49 ; \mathrm{p}=0.02$ ) and a history of sexual relations with HIV carriers (AOR: 7.40; $\mathrm{p}=0.00$ ) were predictors for HIV infection.

Conclusion The results of this study support high rates of risk behaviours for HIV infection among the homeless, evidence of the necessity of actions involving the prevention and treatment of STI on the streets and in temporary and permanent housing. Furthermore, it is extremely important to reach out to intersectoral networks (education, justice, culture and health), with the goal of reintegrating an individual into the job market, supporting the restoration of family and social ties, as well as autonomous living.

\section{P3.189 RECURRENCE OF HUMAN PAPILLOMAVIRUS EXTERNAL GENITAL WART INFECTION AMONG HIGH-RISK ADULTS IN MONTRÉAL, QUÉBEC}

${ }^{1}$ Réjean Thomas, ${ }^{2}$ Marc Steben, ${ }^{3}$ Zoë Greenwald, ${ }^{4}$ Melissa Stutz, ${ }^{5}$ Caroline Rodier ${ }^{5}$ Fern Deangelis, ${ }^{4}$ Emmanouil Rampakakis. ${ }^{1}$ Clinique Médicale L'Actuel, Montreal, Canada; ${ }^{2}$ Institut National De Santé Publique Du Québec, Montreal, Canada; ${ }^{3}$ Clinique Médicale L Actuel, Montreal, Canada; ${ }^{4}$ Jss Medical Research, Montreal, Canada; ${ }^{5}$ Merck Canada, Montreal, Canada

\subsection{6/sextrans-2017-053264.424}

Introduction External genital warts (EGW) are associated with high psychosocial burden and health care costs, may be difficult to treat, and recur frequently. However, the incidence of recurrent EGW is not well characterised; this is especially true in a post-human papilloma virus (HPV) vaccination era. The objectives of this study were to assess the incidence of subsequent HPV-related EGW in high-risk male and female adults, as well as ascertain patient profile and disease characteristics, in a real-world Canadian clinical setting.

Methods A retrospective chart review study was conducted at Clinique médicale l'Actuel, a sexual health clinic in Montréal, Québec, Canada. Eligible patients were 18-45 years of age with a first diagnosis of EGW between July 1, 2006 and June 30, 2012.

Results A total of 400 first-episode EGW cases were identified. Up to 6 subsequent episodes were documented, with 194 (48.5\%) patients reporting at least 1 subsequent EGW episode. Median time to $1^{\text {st }}$ subsequent EGW episode was 3.97 years, and the incidence density rate for all subsequent episodes was $0.18 / 100$ patient-years. Over $90 \%$ of patients reported clearance of the first subsequent episode, with median time to clearance of 0.30 years. Regardless of subsequent episode number, $>95 \%$ of patients received treatment, primarily cryotherapy, with high-risk sexual behaviour reducing as number of episodes increased.

Conclusion Overall, a high rate of subsequent EGW episodes was observed in this high-risk population despite high treatment rates and improvement in high-risk sexual behaviour with increasing number of subsequent episodes. This is the first assessment of the rate of EGW recurrence in a Canadian clinical setting, in addition to proving information related to risk factors, clinical manifestations, and interventions. These data, assessed in a pre-vaccination Québec healthcare system, may be compared to future EGW rates in order to evaluate the impact of a governmentally-funded HPV vaccination program.

Support: R. Thomas has received consulting fees from AbbVie, Gilead, Merck, and ViiV; M. Steben has received speaker honoraria or participated in Advisory Boards for Merck, Paladin, Valeant and Inovio; M. Stutz and E. Rampakakis are both employees of JSS Medical Research; C. Rodier and F. DeAngelis are both employees of Merck Canada Inc. 


\section{P3.190 HIGH RATE OF REPEAT SEXUALLY TRANSMITTED INFECTIONS AMONG MEN WHO HAVE SEX WITH MEN IN SOUTH AFRICA}

${ }^{1}$ Remco Peters, ${ }^{1}$ Oscar Radebe, ${ }^{2}$ Thabo Hamiwe, ${ }^{2}$ Lerato Maboko, ${ }^{1}$ Helen Struthers, ${ }^{1}$ James Mcintyre, ${ }^{2}$ Marleen Kock. 'Anova Health Institute, Johannesburg, South African Republic; ${ }^{2}$ University of Pretoria, Pretoria, South African Republic

\section{$10.1136 /$ sextrans-2017-053264.425}

Introduction Men who have sex with men (MSM) are considered an important key population in South Africa. Insight in the dynamics of sexually transmitted infections (STI) is of paramount importance to address the burden of infection. We aim to characterise incident STI among South African MSM.

Methods This prospective cohort study was conducted at two primary healthcare clinics that specifically target MSM in Johannesburg City Centre and Soweto, South Africa. We recruited MSM presenting with urethral discharge and provided standard syndromic management. Participants were requested to return for follow-up testing after six weeks, or earlier in case of symptoms. A urine specimen and rectal swab were collected for molecular microbiological investigations at both visits.

Results We recruited 78 MSM of whom 69\% identified as gay; $43 \%$ was HIV-infected. Neisseria gonorrhoeae was the main cause of discharge in 46 men (59\%), followed by Chlamydia trachomatis in 9 (12\%), Mycoplasma genitalium in 1 (1.3\%) and Trichomonas vaginalis in 3 (3.8\%). Rectal swabs were positive for $N$. gonorrhoeae (28\%), C. trachomatis (13\%) and $T$. vaginalis $(3.8 \%)$. Sixty-one $(78 \%)$ men came for follow-up visit. Twenty-three men (38\%) had a total of 34 STI diagnosed at follow-up, including 20 urethral and 14 rectal infections. The majority of these infections (68\%) were considered newly acquired STI based on microorganism detected and anatomic site involved. This includes all infections with C. trachomatis $(\mathrm{n}=5), M$. genitalium $(\mathrm{n}=3)$ and $T$. vaginalis $(\mathrm{n}=9)$. Seventeen MSM tested positive for N. gonorrhoeae at follow-up; six of which were new infections.

Conclusion We demonstrate a high rate of repeat STI in this unique cohort of South African MSM and show that most of these infections are newly acquired. This supports the effectiveness of syndromic management for most STI, but also highlights that strengthening of prevention efforts is highly warranted. The observation of repeat $N$. gonorrhoeae infections is concerning and molecular typing for further analysis of these strains is underway.

\section{P3.191 HIV IN INDIGENOUS MSM IN GUATEMALA: A HIDDEN PROBLEM}

Ricardo Mendizabal-Burastero, Ricardo Mendizabal-Burastero, Marco Polo Yancor. Colectivo Amigos Contra El Sida, Guatemala - Guatemala

\subsection{6/sextrans-2017-053264.426}

Introduction In Guatemala, data regarding HIV epidemics doesn't show much data about indigenous population, although nearly $50 \%$ of the population in Guatemala are indigenous. In metropolitan area, MSM HIV prevalence is around 9\%. But there is not data about HIV prevalence in indigenous MSM. This data is the first report in this important group.

Methods Cross-sectional data analysis from October 2015 to July 2016. MSM were recruited as part of the implementation of Global Fund projects. Activities were implemented in three department of the Guatemalan highlands: Sololá, Totonicapan and Chimaltenango. Recruitment was performed by peers, by face-to-face or using social networks. Rapid HIV testing was performed in-site and confirmation in reference laboratory, STI were evaluated by syndromic management. Data was analysed using Stata 13.

Results 1196 MSM were tested for HIV and 293 had STI evaluation. 42\% lived in Chimaltenango and 19\% in Solola. Median age was 22 years old (IQR 19-26); 40.3\% of them self-reported as indigenous, $11 \%$ of them K'iche' and $10 \%$ kakchiquel. $60 \%$ of them had high school or higher education, 58 HIV cases were diagnosed, for a global HIV prevalence of $4.8 \%$. In indigenous MSM HIV prevalence was higher (5.1 vrs 4.9, no difference). In K'iche' MSM (N:156) HIV prevalence was higher (4.2\%) than in kakchiquel $(3.9 \%)$, but no difference. $18 \%$ had an STI, the most prevalent were anal warts $(13 \%)$.

Conclusion HIV prevalence in indigenous MSM is lower than reported in Guatemala city, however is a major public health problem not previously reported in Guatemala. Population representative studies in the highlands are needed to asses HIV prevalence in MSM, as well to improve current interventions.

\section{P3.192 HUMAN PARVOVIRUS B19 AMONG BRAZILIAN PATIENTS INFECTED WITH HIV}

${ }^{1}$ RCN Cubel Garcia, ${ }^{1} \mathrm{KML}$ Azevedo, 's Setubal, ${ }^{2} \mathrm{MM}$ Siqueira, ${ }^{1}$ Oliveira AS. ${ }^{1}$ Federal Fluminense University, Niterói, Brazil; ${ }^{2}$ Oswaldo Cruz Foundation, Rio de Janeiro, RJ, Brazil

\subsection{6/sextrans-2017-053264.427}

Introduction Human Parvovirus B19 (B19V) infection may cause red cell aplasia in patients infected with human immunodeficiency virus (HIV). The introduction of highly active antiretroviral therapy (HAART) has improved the immune function of these patients, modifying the course of B19V infection. To better understand the importance of B19V infection in the HAART era, a follow-up study of a cohort of HIV-infected individuals was conducted during an eight-year period (2001-2008) at the Infectious Diseases Department of Antonio Pedro University Hospital (HUAP) at the Fluminense Federal University (Niterói, RJ, Brazil).

Methods Blood samples were collected from 313 HIV-infected individuals who attended the general medical outpatient clinic for routine care. IgG and IgM antibodies against B19V were detected in serum samples using a commercial enzyme immunoassay (Biotrin). B19V viraemia was evaluated by the detection of B19V-DNA by polymerase chain reaction (PCR). To genotype B19V strains PCR products were subjected to direct sequencing and phylogenetic analysis.

Results The seropositivity to $\mathrm{B} 19 \mathrm{~V}$ IgG antibodies was $72 \%$ (225/313). Approximately 30\% (28/88) of anti-B19V IgG negative patients seroconverted. Most seroconversions occurred during incidence peaks of a B19V infection in Niterói (20052006). No clinical manifestations of B19V infection were detected during the seroconversion period. B19V-DNA was detected in 5/88 patients, four of whom also exhibited seroconversion. Four of the patients were infected with subgenotype 1a strains and the remaining patient was infected with a subgenotype $3 \mathrm{~b}$. Anaemia was detected in $8 / 88$ patients, but 